\title{
SHARP MAXIMAL INEQUALITIES \\ FOR STOCHASTIC INTEGRALS \\ IN WHICH THE INTEGRATOR IS A SUBMARTINGALE
}

\author{
WILLIAM HAMMACK \\ (Communicated by Richard T. Durrett)
}

\begin{abstract}
We obtain sharp maximal inequalities for strong subordinates of real-valued submartingales. Analogous inequalities also hold for stochastic integrals in which the integrator is a submartingale. The impossibility of general moment inequalities is also demonstrated.
\end{abstract}

\section{INTRODUCTION}

Let $(\Omega, \mathcal{F}, P)$ be a complete probability space with a right-continuous filtration $\left(\mathcal{F}_{t}\right)_{t \geq 0}$ where $\mathcal{F}_{0}$ contains all $P$-null sets. Suppose $X$ is an adapted right-continuous real-valued submartingale with left limits and $H$ is a predictable process with values in the closed unit ball of $\mathbb{R}^{\nu}$, where $\nu$ is a positive integer. Define an adapted rightcontinuous process $Y$ with left limits by

$$
Y_{t}=H_{0} X_{0}+\int_{(0, t]} H_{s} d X_{s} .
$$

We will compare the size of $Y$ with that of $X$ by finding constants $\beta$ such that for all $\lambda>0$,

$$
\lambda P\left(Y^{*} \geq \lambda\right) \leq \beta\|X\|_{1}
$$

where $\|X\|_{1}=\sup _{t \geq 0}\left\|X_{t}\right\|_{1}$ and $Y^{*}=\sup _{t \geq 0}\left|Y_{t}\right|$. In this paper we will denote the Euclidean norm of $y \in \mathbb{R}^{\nu}$ by $|y|$ and the inner product of $y, k \in \mathbb{R}^{\nu}$ by $y \cdot k$.

If we restrict $X$ to the class of martingales, it is known that the best constant satisfying (1.1) is $\beta=2[2,3]$. By the best constant we mean that for any $\beta<$ 2 there exist a martingale $X$, a predictable process $H$, and a $\lambda>0$ such that $\lambda P\left(Y^{*} \geq \lambda\right)>\beta\|X\|_{1}$. It is also known [5] that if we restrict $X$ to the class of nonnegative submartingales, then the best constant satisfying (1.1) is $\beta=3$.

In this paper we will show that for the class of real-valued submartingales, the best constant in (1.1) is $\beta=6$. To do this we shall first prove the analogous inequality and more for discrete-time submartingales. In the last section of this

Received by the editors June 15, 1994 and, in revised form, September 20, 1994.

1991 Mathematics Subject Classification. Primary 60G42, 60H05; Secondary 60E15.

Key words and phrases. Martingale, submartingale, maximal inequality, differential subordination, strong subordination, stochastic integral. 
paper we shall show that there are no moment inequalities of the form $\|Y\|_{p} \leq$ $\beta\|X\|_{p}$ where $1<p<\infty$ and $\beta$ is finite and depends only on $p$. In fact, we shall show that for any $p \in[1, \infty)$, there is no finite $\beta$ such that $\|Y\|_{1} \leq \beta\|X\|_{p}$. For the case $p=\infty$, see [7] where it is shown that if $\|X\|_{\infty}=1$, then there is a constant $\gamma$ such that for $\lambda>4, P\left(Y^{*} \geq \lambda\right) \leq \gamma \exp (-\lambda / 4)$, so, for any $r \in[1, \infty),\|Y\|_{r}$ is bounded by some constant depending only on $r$.

\section{A MAXIMAL INEQUALITY FOR SUBMARTINGALES}

Let $f_{0}, f_{1}, \ldots$ be a real-valued submartingale relative to a filtration $\left(\mathcal{F}_{n}\right)_{n \geq 0}$ on a probability space $(\Omega, \mathcal{F}, P)$ with difference sequence $d_{0}, d_{1}, \ldots$, and $g_{0}, g_{1}, \ldots$ an $\mathbb{R}^{\nu}$ valued process adapted to $\left(\mathcal{F}_{n}\right)_{n \geq 0}$ with difference sequence $e_{0}, e_{1}, \ldots$, where $\nu$ is a positive integer. We say that $g$ is strongly subordinate to $f$ if $g$ is both differentially subordinate and conditionally differentially subordinate to $f$, i.e. for all $n \geq 0$, $\left|e_{n}\right| \leq\left|d_{n}\right|$ and $\left|\mathbf{E}\left(e_{n+1} \mid \mathcal{F}_{n}\right)\right| \leq\left|\mathbf{E}\left(d_{n+1} \mid \mathcal{F}_{n}\right)\right|$. Note that if for $k \geq 0, e_{k}=h_{k} d_{k}$ where $h_{k}: \Omega \rightarrow[-1,1]$ is $\mathcal{F}_{k-1}$-measurable, then $g$ is strongly subordinate to $f$. In particular, if $g$ is a \pm 1 -transform of $f$, i.e. $e_{k}=\epsilon_{k} d_{k}$ where $\epsilon_{k} \in\{-1,1\}$, then $g$ is strongly subordinate to $f$.

Theorem 2.1. If $f=\left(f_{n}\right)_{n \geq 0}$ is a submartingale relative to a filtration $\left(\mathcal{F}_{n}\right)_{n \geq 0}$ and $g=\left(g_{n}\right)_{n \geq 0}$ is strongly subordinate to $f$, then for all $\lambda>0$,

$$
\lambda P\left(g^{*} \geq \lambda\right) \leq \lambda P\left(\sup _{n \geq 0}\left(\left|f_{n}\right|+\left|g_{n}\right|\right) \geq \lambda\right) \leq 4 \sup _{n \geq 0} \mathbf{E} f_{n}^{+}-2 \mathbf{E} f_{0}
$$

where $g^{*}=\sup _{n \geq 0}\left|g_{n}\right|$.

Remarks. If $f$ is a martingale, then $\mathbf{E} f_{n}^{+}$and $\mathbf{E} f_{n}^{-}$are nondecreasing sequences. It then follows from $\mathbf{E} f_{0}=\mathbf{E} f_{n}^{+}-\mathbf{E} f_{n}^{-}$that $\|f\|_{1}=2 \sup _{n \geq 0} \mathbf{E} f_{n}^{+}-\mathbf{E} f_{0}$, where $\|f\|_{1}=\sup _{n \geq 0}\left\|f_{n}\right\|_{1}$. Thus in the martingale case, (2.1) implies that

$$
\lambda P\left(g^{*} \geq \lambda\right) \leq \lambda P\left(\sup _{n \geq 0}\left(\left|f_{n}\right|+\left|g_{n}\right|\right) \geq \lambda\right) \leq 2\|f\|_{1}
$$

which is Theorem 4.1 of [4]. If $f$ is a nonnegative supermartingale, (2.1) implies

$$
\lambda P\left(g^{*} \geq \lambda\right) \leq \lambda P\left(\sup _{n \geq 0}\left(\left|f_{n}\right|+\left|g_{n}\right|\right) \geq \lambda\right) \leq 2 \mathbf{E} f_{0}
$$

which is Theorem 8.1 of [5]. Both results are shown to be sharp in the articles quoted. If $f$ is a nonnegative submartingale with $f_{0}=0$, the resulting inequality is not sharp in the case $f_{0}=0$, as can be seen from Theorem 4.1 of [5] which shows in this case

$$
\lambda P\left(g^{*} \geq \lambda\right) \leq \lambda P\left(\sup _{n \geq 0}\left(\left|f_{n}\right|+\left|g_{n}\right|\right) \geq \lambda\right) \leq 3\|f\|_{1} .
$$

Proof. We will assume $\|f\|_{1}$ is finite. This is equivalent to saying $\sup _{n \geq 0} \mathbf{E} f_{n}^{+}$is finite, as for all $n \geq 0, \mathbf{E} f_{n}^{+} \leq\left\|f_{n}\right\|_{1} \leq 2 \mathbf{E} f_{n}^{+}-\mathbf{E} f_{0}$. The first inequality is obvious, the second follows from $\mathbf{E} f_{0} \leq \mathbf{E} f_{n}=\mathbf{E} f_{n}^{+}-\mathbf{E} f_{n}^{-}$.

To show (2.1), it suffices to show that for $n \geq 0$,

$$
\lambda P\left(\left|f_{n}\right|+\left|g_{n}\right| \geq \lambda\right) \leq 4 \mathbf{E} f_{n}^{+}-2 \mathbf{E} f_{0}
$$


since if (2.2) holds, then with $\tau=\inf \left\{n \geq 0:\left|f_{n}\right|+\left|g_{n}\right| \geq \lambda\right\}, \tau$ is a stopping time, $f^{\tau}$ is a submartingale, and $g^{\tau}$ is strongly subordinate to $f^{\tau}$, so by $(2.2)$

$$
\lambda P\left(\sup _{m \leq n}\left(\left|f_{m}\right|+\left|g_{m}\right|\right) \geq \lambda\right)=\lambda P\left(\left|f_{\tau \wedge n}\right|+\left|g_{\tau \wedge n}\right| \geq \lambda\right) \leq 4 \mathbf{E} f_{\tau \wedge n}^{+}-2 \mathbf{E} f_{0} .
$$

Since $\left(f_{n}^{+}\right)_{n \geq 0}$ is a submartingale, it follows by Doob's optional sampling theorem that $\mathbf{E} f_{\tau \wedge n}^{+} \leq \mathbf{E} f_{n}^{+}$, thus implying (2.1).

By dividing by $\lambda$ throughout in (2.2), we may assume $\lambda=1$. Using the methods developed by Burkholder [2], we define $V$ on $\mathbb{R} \times \mathbb{R}^{\nu}$ by

$$
V(x, y)= \begin{cases}1-4 x^{+}, & \text {if }|x|+|y| \geq 1 \\ -4 x^{+}, & \text {if }|x|+|y|<1\end{cases}
$$

Then (2.2) is equivalent to $\mathbf{E} V\left(f_{n}, g_{n}\right) \leq-2 \mathbf{E} f_{0}$. Define $U$ on $\mathbb{R} \times \mathbb{R}^{\nu}$ by

$$
U(x, y)= \begin{cases}1-4 x^{+}, & \text {if }|x|+|y| \geq 1 \\ |y|^{2}-x^{2}-2 x, & \text { if }|x|+|y|<1\end{cases}
$$

Then $V \leq U$ (in the case of $|x|+|y|<1$ this follows from $-4 x^{+} \leq-x^{2}-2 x$ for $|x|<1$ ) and $U\left(f_{0}, g_{0}\right) \leq-2 f_{0}$ (recall that by assumption $\left|f_{0}\right| \geq\left|g_{0}\right|$ ).

Thus $\mathbf{E} V\left(f_{n}, g_{n}\right) \leq \mathbf{E} U\left(f_{n}, g_{n}\right)$ and $\mathbf{E} U\left(f_{0}, g_{0}\right) \leq-2 \mathbf{E} f_{0}$. To show (2.2), it will suffice to show that for $1 \leq j \leq n$,

$$
\mathbf{E} U\left(f_{j}, g_{j}\right) \leq \mathbf{E} U\left(f_{j-1}, g_{j-1}\right) .
$$

Define $\phi, \psi$ on $\mathbb{R} \times \mathbb{R}^{\nu}$ by

$$
\begin{aligned}
& \phi(x, y)= \begin{cases}-4, & \text { if }|x|+|y| \geq 1 \text { and } x \geq 0 \\
0, & \text { if }|x|+|y| \geq 1 \text { and } x<0 \\
-2 x-2, & \text { if }|x|+|y|<1,\end{cases} \\
& \psi(x, y)= \begin{cases}0, & \text { if }|x|+|y| \geq 1, \\
2 y, & \text { if }|x|+|y|<1 .\end{cases}
\end{aligned}
$$

Then $U_{x}(x, y)=\phi(x, y)$ and $U_{y}(x, y)=\psi(x, y)$ for $|x|+|y| \neq 1, y \neq 0$, and $x \neq 0$ where $U_{x}(x, y)$ and $U_{y}(x, y)$ are the partials of $U$ with respect to $x$ and $y$ respectively. Note that $|\psi| \leq-\phi$.

Claim: Given $h \in \mathbb{R}$ and $k \in \mathbb{R}^{\nu}$ with $|k| \leq|h|$, then for all $x \in \mathbb{R}$ and $y \in \mathbb{R}^{\nu}$

$$
U(x+h, y+k) \leq U(x, y)+\phi(x, y) h+\psi(x, y) \cdot k .
$$

This can be verified by checking the various cases:

For $|x|+|y| \geq 1$ and $x \geq 0$, we need to show $U(x+h, y+k) \leq 1-4(x+h)$. For $|x+h|+|y+k| \geq 1$ this is clear. For $|x+h|+|y+k|<1$ it follows from

$$
|y+k|^{2}<(1-|x+h|)^{2} \leq 1-2(x+h)+(x+h)^{2} .
$$

For $|x|+|y| \geq 1$ and $x<0$, we need to show $U(x+h, y+k) \leq 1$. However $U(x, y) \leq 1$ for all $x, y$, this being obvious for $|x|+|y| \geq 1$. In the region $|x|+|y|<1$, since $U_{x}(x, y) \leq 0$, it follows that $U(x, y) \leq|y|^{2}-(|y|-1)^{2}-2(|y|-1)=1$. 
For the case $|x|+|y|<1,(2.4)$ is equivalent to showing

$$
U(x+h, y+k) \leq|y+k|^{2}-(x+h)^{2}-2(x+h)-|k|^{2}+h^{2} .
$$

For $|x+h|+|y+k|<1$, this follows from $|k| \leq|h|$ and the definition of $U$. For $|x+h|+|y+k| \geq 1,(2.5)$ can be rewritten as

$$
(1-|x+h|)^{2} \leq|y+k|^{2}+h^{2}-|k|^{2} .
$$

If $|x+h| \leq 1$, this inequality follows from $|k| \leq|h|$ and $|x+h|+|y+k| \geq 1$. If $|x+h|>1$, then $(1-|x+h|)^{2} \leq(1-|x|-|h|)^{2}$ and it suffices to show

$$
(1-|x|)^{2}-2|h|(1-|x|) \leq|y|^{2}-2|y||k| .
$$

Since $|h| \geq|k|$, it then suffices to show $(1-|x|)^{2} \leq|y|^{2}+2|h|(1-|x|-|y|)$, an inequality which follows from $|x|+|h| \geq 1$ and $0 \leq|y|^{2}-2|y|(1-|x|)+(1-|x|)^{2}$, so that

$$
(1-|x|)^{2} \leq|y|^{2}+2(1-|x|)(1-|x|-|y|) \leq|y|^{2}+2|h|(1-|x|-|y|) .
$$

To prove (2.3), since $\left|e_{j}\right| \leq\left|d_{j}\right|$, by (2.4) we have

$$
U\left(f_{j}, g_{j}\right) \leq U\left(f_{j-1}, g_{j-1}\right)+\phi\left(f_{j-1}, g_{j-1}\right) d_{j}+\psi\left(f_{j-1}, g_{j-1}\right) \cdot e_{j} .
$$

Since $f$ is a submartingale, $\mathbf{E}\left(d_{j} \mid \mathcal{F}_{j-1}\right) \geq 0$. It then follows from $|\psi| \leq-\phi$ and $g$ being strongly subordinate to $f$ that

$$
\phi\left(f_{j-1}, g_{j-1}\right) \mathbf{E}\left(d_{j} \mid \mathcal{F}_{j-1}\right)+\psi\left(f_{j-1}, g_{j-1}\right) \cdot \mathbf{E}\left(e_{j} \mid \mathcal{F}_{j-1}\right) \leq 0 .
$$

Using this after taking the conditional expectations relative to $\mathcal{F}_{j-1}$ in (2.6) gives

$$
\mathbf{E}\left(U\left(f_{j}, g_{j}\right) \mid \mathcal{F}_{j-1}\right) \leq U\left(f_{j-1}, g_{j-1}\right) .
$$

Taking expectations of both sides gives (2.3) and completes the proof.

\section{Discrete-time Sharp MAXimal inequalities}

Theorem 3.1. If $f$ is a submartingale relative to a filtration $\left(\mathcal{F}_{n}\right)_{n \geq 0}$ and $g$ is strongly subordinate to $f$, then for all $\lambda>0$

$$
\lambda P\left(g^{*} \geq \lambda\right) \leq \lambda P\left(\sup _{n \geq 0}\left(\left|f_{n}\right|+\left|g_{n}\right|\right) \geq \lambda\right) \leq 4\|f\|_{1}-2 \mathbf{E} f_{0} .
$$

Thus if $f_{0} \equiv 0$, then

$$
\lambda P\left(g^{*} \geq \lambda\right) \leq \lambda P\left(\sup _{n \geq 0}\left(\left|f_{n}\right|+\left|g_{n}\right|\right) \geq \lambda\right) \leq 4\|f\|_{1},
$$

while in general

$$
\lambda P\left(g^{*} \geq \lambda\right) \leq \lambda P\left(\sup _{n \geq 0}\left(\left|f_{n}\right|+\left|g_{n}\right|\right) \geq \lambda\right) \leq 6\|f\|_{1} .
$$

The constants 4 and 6 are the best possible in (3.2) and (3.3) respectively, even in the case $\nu=1$ and $g$ is a \pm 1 -transform of $f$.

Proof. The inequalities follow immediately from Theorem 2.1. For the sharpness, first consider the following example: 
Example 3.1. Fix $3<\beta<4$ and let $\alpha=(4-\beta) / 4$, so that $\beta<4-2 \alpha$. On the Lebesgue interval $[0,1]$, let $f_{0}=g_{0} \equiv 0$,

$$
\begin{array}{cc}
f_{1}=\mathbf{1}_{[0, \alpha)}-\frac{\alpha}{1-\alpha} \mathbf{1}_{[\alpha, 1]}, & g_{1}=f_{1}, \\
f_{2}=f_{1}-\mathbf{1}_{\left[\alpha, 2 \alpha-\alpha^{2}\right)}+\frac{\alpha}{1-\alpha} \mathbf{1}_{\left[2 \alpha-\alpha^{2}, 1\right]}, & g_{2}=g_{1}+\mathbf{1}_{\left[\alpha, 2 \alpha-\alpha^{2}\right)}-\frac{\alpha}{1-\alpha} \mathbf{1}_{\left[2 \alpha-\alpha^{2}, 1\right]}, \\
f_{3}=f_{2}+\frac{1}{1-\alpha} \mathbf{1}_{\left[\alpha, 2 \alpha-\alpha^{2}\right)}, & g_{3}=g_{2}+\frac{1}{1-\alpha} \mathbf{1}_{\left[\alpha, 2 \alpha-\alpha^{2}\right)} .
\end{array}
$$

Then $f=\left(f_{0}, f_{1}, f_{2}, f_{3}\right)$ is a submartingale and $g=\left(g_{0}, g_{1}, g_{2}, g_{3}\right)$ is a \pm 1 transform of $f$. Note that $g_{3}=\mathbf{1}_{[0, \alpha)}+2 \mathbf{1}_{\left[\alpha, 2 \alpha-\alpha^{2}\right)}-(2 \alpha /(1-\alpha)) \mathbf{1}_{\left[2 \alpha-\alpha^{2}, 1\right]}$ and $f_{3}=\mathbf{1}_{[0, \alpha)}$. Thus

$$
2 P\left(f_{3}+g_{3} \geq 2\right)=(4-2 \alpha) \alpha>\beta \sup _{0 \leq j \leq 3} \mathbf{E} f_{j}^{+}
$$

Now let $\tilde{f}_{0}=\tilde{g}_{0} \equiv 0$ and for $j \geq 0,1 \leq k \leq 3$, and $s \in[0,1]$, let

$$
\begin{aligned}
& \tilde{f}_{3 j+k}(s)=\tilde{f}_{3 j}(s)+\mathbf{1}_{\left[1-2^{-j}, 1-2^{-j-1}\right]}(s) f_{k}\left(2^{j+1}\left(s-1+2^{-j}\right)\right), \\
& \tilde{g}_{3 j+k}(s)=\tilde{g}_{3 j}(s)+\mathbf{1}_{\left[1-2^{-j}, 1-2^{-j-1}\right]}(s) g_{k}\left(2^{j+1}\left(s-1+2^{-j}\right)\right) .
\end{aligned}
$$

By induction on $j \geq 0$, we have

$$
\begin{gathered}
P\left(\tilde{f}_{3 j}=1, \tilde{g}_{3 j}=1\right)=\left(1-2^{-j}\right) \alpha, \quad P\left(\tilde{f}_{3 j}=0, \tilde{g}_{3 j}=2\right)=\left(1-2^{-j}\right)\left(\alpha-\alpha^{2}\right), \\
P\left(\tilde{f}_{3 j}=0, \tilde{g}_{3 j}=\frac{-2 \alpha}{1-\alpha}\right)=\left(1-2^{-j}\right)(1-\alpha)^{2},
\end{gathered}
$$

and, for $k \leq 3 j, \operatorname{supp} \tilde{f}_{k} \subseteq\left[0,1-2^{-j}\right]$.

It follows that $\tilde{f}$ is a submartingale, $\tilde{g}$ is a \pm 1 -transform of $f$, and, for $j \geq 0$, $1 \leq k \leq 3,\left\|\tilde{f}_{3 j+k}\right\|_{1}=\left\|\tilde{f}_{3 j}\right\|_{1}+2^{-j-1}\left\|f_{k}\right\|_{1}$. Since $\left\|\tilde{f}_{3 j}\right\|_{1}=\left(1-2^{-j}\right) \alpha$ and $\left\|f_{1}\right\|_{1}=\left\|f_{2}\right\|_{1}=2 \alpha$, we have that $\left\|\tilde{f}_{3 j+k}\right\|_{1} \leq \alpha=\mathbf{E} f_{3}$. Thus, with $\lambda=2$,

$$
\lim _{j \rightarrow \infty} \lambda P\left(\tilde{f}_{3 j}+\tilde{g}_{3 j} \geq \lambda\right)=\lambda P\left(f_{3}+g_{3} \geq \lambda\right)>\beta \mathbf{E} f_{3} \geq \beta \sup _{k \geq 0}\left\|\tilde{f}_{k}\right\|_{1} .
$$

Since we are assuming a strict inequality, there exists an $n$ such

$$
\lambda P\left(\tilde{f}_{n}+\tilde{g}_{n} \geq \lambda\right)>\beta \sup _{j \geq 0}\left\|\tilde{f}_{j}\right\|_{1} \geq \beta \sup _{0 \leq j \leq n}\left\|\tilde{f}_{j}\right\|_{1} .
$$

Now let $\left(r_{j}\right)_{j \geq 1}$ be a sequence of independent identically distributed random variables such that $P\left(r_{1}=1\right)=P\left(r_{1}=-1\right)=\frac{1}{2}$ and the $\left(r_{j}\right)$ are independent from both the $\left(\tilde{f}_{j}\right)$ and the $\left(\tilde{g}_{j}\right)$

For $j \geq 0$, let $\tilde{f}_{n+j+1}=\tilde{f}_{n+j}+\tilde{f}_{n+j} r_{j+1}$ and $\tilde{g}_{n+j+1}=\tilde{g}_{n+j}-\tilde{f}_{n+j} r_{j+1}$. By this sequence of double or nothings we have that for $j \geq n,\left\|\tilde{f}_{j}\right\|_{1}=\left\|\tilde{f}_{n}\right\|_{1}$, yet

$$
\lim _{m \rightarrow \infty} \lambda P\left(\tilde{g}_{m} \geq \lambda\right)=\lambda P\left(\tilde{f}_{n}+\tilde{g}_{n} \geq \lambda\right)>\beta\|\tilde{f}\|_{1}
$$


and since we are assuming a strict inequality, we can choose an $m>n$ that satisfies

$$
\lambda P\left(\tilde{g}_{m} \geq \lambda\right)>\beta\|\tilde{f}\|_{1}
$$

This immediately implies the sharpness in (3.2). To show the sharpness in (3.3), it suffices to use $\tilde{f}$ and $\tilde{g}$ to construct a submartingale $F$ with a \pm 1 -transform $G$ such that

$$
\lambda P\left(\sup _{j \geq 0} G_{j} \geq \lambda\right)>\frac{3}{2} \beta\|F\|_{1} .
$$

Let $\alpha=P\left(\sup _{0 \leq j \leq m} \tilde{g}_{j} \geq \lambda\right)$ so that $\alpha>0$ and let $\delta=\left(4\|\tilde{f}\|_{1}-\lambda \alpha\right) /(6-6 \alpha)$ (in the case $\alpha=1$, let $\delta=0$ ). By (3.2), $\lambda \alpha \leq 4\|\tilde{f}\|_{1}$, hence $\delta \geq 0$.

Let $s$ and $t$ be independent random variables, independent from the $\left(\tilde{f}_{j}\right)$ such that $P(s=\lambda / 6)=\alpha$ and $P(s=\delta)=1-\alpha$, while $P(t=-1)=2 / 3$ and $P(t=2)=1 / 3$. Note that $\mathbf{E} s \leq 2\|\tilde{f}\|_{1} / 3$.

Let $F_{0}=-s, G_{0}=s, F_{1}=F_{0}+t F_{0}$, and $G_{1}=G_{0}-t F_{0}$. We then have that $\left\|F_{1}\right\|_{1}=\left\|F_{0}\right\|_{1}=\mathbf{E} s$.

Let $F_{2}=F_{1}-F_{1}$ and $G_{2}=G_{1}-F_{1}$. Thus $F_{2}=0$ a.s. while $G_{2}=6 s$ on the set $\{t=2\}$ and $G_{2}=0$ on the set $\{t=-1\}$. We then have that

$$
\begin{gathered}
P\left(F_{2}=0, G_{2}=\lambda\right)=\alpha / 3, \quad P\left(F_{2}=0, G_{2}=6 \delta\right)=(1-\alpha) / 3, \\
P\left(F_{2}=0, G_{2}=0\right)=2 / 3 .
\end{gathered}
$$

Let $A=\left\{G_{2}=0\right\}$ and, for $j \geq 1$, let $F_{2+j}=\mathbf{1}_{A} \tilde{f}_{j}$ and $G_{2+j}=G_{2}+\mathbf{1}_{A} \tilde{g}_{j}$. Then by the independence of $t$ and the $\left(\tilde{f}_{j}\right), F$ is a submartingale, $G$ is a \pm 1 -transfrom of $F$, and for $j \geq 1$ we have that $\left\|F_{2+j}\right\|_{1}=2\left\|\tilde{f}_{j}\right\|_{1} / 3$, while

$$
\begin{aligned}
P\left(\sup _{0 \leq j \leq m+2} G_{j} \geq \lambda\right) & =P\left(\sup _{0 \leq j \leq 2} G_{j} \geq \lambda\right)+\frac{2}{3} P\left(\sup _{0<j \leq m} \tilde{g}_{j} \geq \lambda\right) \\
& \geq \frac{1}{3} \alpha+\frac{2}{3} P\left(\sup _{0<j \leq m} \tilde{g}_{j} \geq \lambda\right)=P\left(\sup _{0 \leq j \leq m} \tilde{g}_{j} \geq \lambda\right)
\end{aligned}
$$

so that

$$
\lambda P\left(\sup _{0 \leq j \leq m+2} G_{j} \geq \lambda\right) \geq \lambda P\left(\sup _{0 \leq j \leq m} \tilde{g}_{j} \geq \lambda\right)>\beta\|\tilde{f}\|_{1} \geq \frac{3 \beta}{2}\|F\|_{1}
$$

\section{Applications to stochastic integrals}

Theorem 4.1. With $(\Omega, \mathcal{F}, P)$ and $\left(\mathcal{F}_{t}\right)_{t \geq 0}$ as in Section 1 , suppose $X$ is an adapted right-continuous submartingale with left limits such that $\mathbf{E} X_{0}$ is finite and $H$ is a predictable process with values in the closed unit ball of $\mathbb{R}^{\nu}$. Then with $Y$ defined by $Y_{t}=H_{0} X_{0}+\int_{(0, t]} H_{s} d X_{s}$, we have that, for $\lambda>0$,

$$
\lambda P\left(Y^{*} \geq \lambda\right) \leq 4 \sup _{t \geq 0} \mathbf{E} X_{t}^{+}-2 \mathbf{E} X_{0}
$$


so that

$$
\lambda P\left(Y^{*} \geq \lambda\right) \leq 6\|X\|_{1}
$$

and if $X_{0} \equiv 0$, then

$$
\lambda P\left(Y^{*} \geq \lambda\right) \leq 4\|X\|_{1}
$$

The constants 6 and 4 in (4.2) and (4.3) respectively are the best possible.

Proof. As in Theorem 2.1 we have $\mathbf{E} X_{t}^{+} \leq\left\|X_{t}\right\|_{1} \leq 2 \mathbf{E} X_{t}^{+}-\mathbf{E} X_{0}$, hence we can assume the finiteness of $\|X\|_{1}$. The proof follows in the same way as the proof of Theorem 5.1 of [5], except that we use Theorem 2.1 above to show that $X$ is an $L^{1, \infty}$-integrator in the sense of [1].

The sharpness in (4.2) and (4.3) follow from the sharpness in (3.8) and (3.7) holding even for \pm 1 -transforms.

\section{LACK OF $L^{p}$ INEQUALITIES}

Fix $p \in[1, \infty)$ and $\beta>1$. We shall construct a discrete time submartingale $F=\left(F_{0}, F_{1}, \ldots\right)$ with $F_{0}=0$ and a \pm 1 -transform of $F, G=\left(G_{0}, G_{1}, \ldots\right)$ such that

$$
\|G\|_{1}>\beta\|F\|_{p}
$$

To do this, we will first construct a finite length submartingale $f=\left(f_{0}, f_{1}, \ldots, f_{N}\right)$ with $f_{0}=0$, and $f_{N} \geq 0$ together with a \pm 1 -transform of $f, g=\left(g_{0}, \ldots, g_{N}\right)$ such that

$$
\|g\|_{1}>\beta\left\|f^{+}\right\|_{p}
$$

where $\left\|f^{+}\right\|_{p}=\sup _{0 \leq n \leq N} \mathbf{E}\left(f_{n}^{+}\right)^{p}$. Let $M>4 \beta$ and let $\left(r_{1}, \ldots, r_{2 M}\right)$ be a sequence of independent random variables such that for $j=1,2, P\left(r_{j}=1\right)=P\left(r_{j}=-1\right)=$ $1 / 2$ and for $2 \leq j \leq M$,

$$
\begin{gathered}
P\left(r_{2 j-1}=1\right)=P\left(r_{2 j-1}=-1\right)=\frac{1}{2}, \\
P\left(r_{2 j}=-1\right)=\frac{1}{3}, \quad P\left(r_{2 j}=\frac{1}{2}\right)=\frac{2}{3} .
\end{gathered}
$$

Let $f_{j}=\sum_{k=0}^{j} d_{k}$ where $d_{0}=0, d_{1}=r_{1} / 2, d_{2}=r_{2} / 2$, and $d_{j}=\mathbf{1}_{\left\{f_{j-1}<0\right\}} r_{j} f_{j-1}$ for $j>2$. By the independence of the $r_{j},\left(f_{j}\right)_{j \leq 2 M}$ forms a martingale. Note that for $j \geq 1$,

$$
\begin{gathered}
P\left(f_{2 j}=1\right)=\frac{1}{4}, \quad P\left(f_{2 j}=-3^{j-1}\right)=\frac{1}{4}\left(\frac{1}{3}\right)^{j-1}, \\
P\left(f_{2 j}=0\right)=\frac{3}{4}-\frac{1}{4}\left(\frac{1}{3}\right)^{j-1} .
\end{gathered}
$$


For $0 \leq j \leq 2 M$, let $g_{j}=\sum_{k=0}^{j}(-1)^{k} d_{k}$. Then for $2 \leq j \leq 2 M,\left\|f_{j}^{+}\right\|_{p}^{p}=$ $\left\|f_{j}^{-}\right\|_{1}=1 / 4$, while for $j \geq 1$,

$$
\left\|g_{2 j+2}\right\|_{1}=\left\|g_{2 j+1}\right\|_{1}=\left\|g_{2 j}\right\|_{1}+1 / 4 \text {. }
$$

Since $\left\|g_{2}\right\|_{1}=1 / 2$, it follows that $\left\|g_{2 M}\right\|_{1}=(M+1) / 4$. Now let $N=2 M+1$, $f_{N}=f_{2 M}^{+}$, and $g_{N}=g_{2 M}+\mathbf{1}_{\left\{f_{2 M}<0\right\}}\left|f_{2 M}\right|$. Then $f=\left(f_{0}, \ldots, f_{N}\right)$ forms a submartingale, $\left\|f^{+}\right\|_{p}^{p}=1 / 4$, and, since $f_{2 M}<0$ implies $g_{2 M}=0,\left\|g_{N}\right\|_{1} \geq$ $\left\|g_{2 M}\right\|_{1}-\left\|f_{2 M}^{-}\right\|_{1}=M / 4>\beta\left\|f^{+}\right\|_{p}$ by our choice of $M$.

To construct $F$ and $G$, we will work with only a small portion of the probability space at a time in order to keep $\|F\|_{p}$ close to that of $\left\|f^{+}\right\|_{p}$. More explicitly, by enriching the probability space if necessary, let $A_{1}, \ldots, A_{K}$ be a partition of the space such that $\sigma\left(A_{1}, \ldots, A_{K}\right)$ is independent of $\sigma\left(f_{0}, \ldots, f_{N}\right)$ and, for $1 \leq j \leq k$, $P\left(A_{j}\right) \leq \epsilon / 3^{M p}$, where $\epsilon$ satisfies $\beta^{p}\left(\left\|f^{+}\right\|_{p}^{p}+\epsilon\right)<\left\|g_{N}\right\|_{1}^{p}$.

Let $F_{0}=G_{0}=0$ and for $1 \leq k \leq K$ and $1 \leq n \leq N$, let

$$
F_{(k-1) N+n}=F_{(k-1) N}+\mathbf{1}_{A_{k}} f_{n}, \quad G_{(k-1) N+n}=G_{(k-1) N}+\mathbf{1}_{A_{k}} g_{n} .
$$

Then $F$ is a submartingale and $G$ is a \pm 1 -transform of $F$. Since $A_{1}, \ldots, A_{N}$ partition the space, $G_{K N}=g_{N}$ and for $1 \leq k \leq K$ and $1 \leq n \leq N$, the disjointness of the $A_{j}$ gives us

$$
\left\|F_{(k-1) N+n}\right\|_{p}^{p}=\left\|f_{N} \mathbf{1}\left(\bigcup_{j=1}^{k-1} A_{j}\right)\right\|_{p}^{p}+\left\|f_{n} \mathbf{1}_{A_{k}}\right\|_{p}^{p} .
$$

Since $f_{N} \geq 0$ a.s. and the $f_{j}$ are bounded in absolute value by $3^{M}$, we have that

$$
\left\|F_{(k-1) N+n}\right\|_{p}^{p} \leq\left\|f_{N}^{+}\right\|_{p}^{p}+3^{M p} P\left(A_{k}\right) \leq\left\|f^{+}\right\|_{p}^{p}+\epsilon
$$

which gives us (5.1) by our choice of $\epsilon$.

\section{ACKNOWLEDGMENT}

The author is grateful to Donald Burkholder for his advice and criticisms.

\section{REFERENCES}

1. K. Bichteler, Stochastic integration and $L^{p}$-theory of semimartingales, Ann. Probab. 9 (1981), 49-89. MR 82g:60071

2. D. L. Burkholder, Boundary value problems and sharp inequalities for martingale transforms, Ann. Probab. 12 (1984), 647-702. MR 86b:60080

3. Sharp inequalities for martingales and stochastic integrals, Astérisque 157 (1988), 75-94. MR 90b:60051

4. , Differential subordination of harmonic functions and martingales, Proceedings of the Seminar on Harmonic Analysis and Partial Differential Equations, El Escorial, 1987, Lecture Notes in Math., vol. 1384, Springer-Verlag, New York, 1989, pp. 1-23. MR 90k:31004

5. Strong differential subordination and stochastic integration, Ann. Probab. 22 (1994), 995-1025. CMP 94:16

6. C. Dellacherie and P. Meyer, Probabilities and potentials B, North-Holland, Amsterdam, 1982. MR 85e:60001

7. W. Hammack, Sharp inequalities for the distribution of a stochastic integral in which the integrator is a bounded submartingale, Ann. Probab. 23 (1995), 223-235.

Department of Mathematics, University of British Columbia, Vancouver, British Columbia, Canada V6T 1Z2

E-mail address: hammack@math.ubc.ca 